\title{
Study Design Configuration Code
}

National Cancer Institute

\section{Source}

National Cancer Institute. Study Design Configuration Code. NCI Thesaurus. Code C93678.

A coded value specifying a trial pattern developed to compare treatment groups in a clinical pre-clinical trial. 